\title{
Über die Vorzüge praktischer Primärsammlungen für die Erschließung ihrer Inhalte, dargestellt an den Dresdner Musikquellen
}

Als Hauptcharakteristikum der historischen Musikbestände in der heutigen Sächsischen Landesbibliothek - Staats- und Universitätsbibliothek (SLUB) Dresden ist ihr Gewachsensein am Ort ihrer jeweiligen praktischen Nutzung anzusehen. Das gilt für die originär Dresdner Sammlungen wie auch für die von auswärts stammenden. Sekundärsammlungen privaten Ursprungs oder nachträgliche Einzelerwerbungen spielen ihnen gegenüber eine marginale Rolle. So vermitteln die vorliegenden Bestände ein Bild vom Musikleben ihrer Entstehungszeit in unterschiedlichen Gesellschaftskreisen und auf unterschiedlichem praktischem Niveau, und darin liegt ihre wesentlichste Bedeutung.

Festzustellen ist dabei allerdings, dass der heutige Besitz der Dresdner Musikabteilung sich zu demjenigen, den sie eigentlich hätte umfassen können, etwa so verhält wie ein Mittelgebirge zu einem Hochgebirge, also z. B. wie das Erzgebirge zu den Alpen. Was in der Erdgeschichte teils durch Naturkatastrophen, teils durch die Atmosphärilien bewirkt wurde, nämlich einerseits durch gewaltsame Zerstörung und andererseits durch allmähliche Erosion (Abtragung und Auswaschung), das bewerkstelligten in der Musiküberlieferung Kriege und Brände bzw. Verschleiß und Achtlosigkeit.

Indessen zeigt sich das heutige ,Erzgebirge 'Dresdner Musiksammlungen noch immer imposant und vielseitig mit stattlichen, weithin sichtbaren Erhebungen. Dass dies so ist, beruht auf einer Reihe von Beständen, die in corpore von ihrem Ursprungsort in die Königliche bzw. Landesbibliothek gelangten und zuvor der praktischen Nutzung gedient hatten. Hervorzuheben sind

- Sammlungen aus sächsischen Stadtschulen und Stadtkirchen (überwiegend Stimmen-Konvolute, häufig mit gedruckten und handschriftlichen Anteilen):

- Annaberg, Stadtkirche (zwei bedeutende Chorbücher aus der ersten Hälfte des 16. Jahrhunderts),

- Grimma, Fürstenschule (evangelische Kirchenmusik aus dem späten 16. bis zum 19. Jahrhundert in bedeutendem Umfang),

- Löbau, Ratsbibliothek (evangelische Kirchenmusik aus dem 16. und 17. Jahrhundert), 
- Pirna (Chorbücher aus dem 16. Jahrhundert und Sammelstimmen vorwiegend aus dem 17. Jahrhundert),

- Schellenberg (evangelische Kirchenmusik aus dem 17. Jahrhundert);

- Sammlungen stadtbürgerlicher Herkunft:

- Zittau, Sammlung Exner (Instrumental-, vor allem Orchestermusik des späten 18. und frühen 19. Jahrhunderts mit Schwerpunkt auf der Wiener Vor- und Hochklassik, zusammengetragen vom Zittauer Handelsherrn Christian August Exner und genutzt für dessen öffentliche Konzerte),

- Dresden, Dreyßigsche Singakademie (19.Jahrhundert; nach Auflösung der Chorvereinigung lückenhaft an die Sächsische Landesbibliothek gelangte Dirigierpartituren),

- und die Sammlung des Herzogs Friedrich August von Braunschweig-Oels (mit der gesamten Bibliothek dieses Fürsten - Lebenszeit 1740-1805 - im 19. Jahrhundert per Erbschaft aus Oels in Schlesien an das sächsische Königshaus gekommen und der Königlichen Öffentlichen Bibliothek direkt oder mit Umweg über die Königliche Privat-Musikaliensammlung einverleibt).

Alle diese Sammlungen besitzen ihren besonderen Wert nicht nur durch ihre Zeugenschaft für praktische Musiziertraditionen bestimmter Institutionen kirchlicher und weltlicher Art, sondern auch durch den historischen Kontext, der, soweit gedruckt vorliegend, in der SLUB greifbar ist (Saxonica- und Silesia-Bestände), ansonsten in der handschriftlichen Überlieferung der zuständigen Stadtarchive recherchiert werden kann.

Die Sammlungen von evangelischer Kirchenmusik greifen inhaltlich ineinander und vermitteln ein vielseitiges Bild von der ehemaligen Verbreitung der durch sie überlieferten Kompositionen. Die Musikalien aus Zittau und aus Dresden (Dreyßigsche Singakademie) gewähren geradezu fotografische Einblicke in bürgerliches Engagement von Dilettanten (im wörtlichen Sinne von „Liebhabern“), die Oelser Erbschaft hingegen in die Möglichkeiten, die ein überaus engagierter Klein-Fürst in der Provinz mit nur teilweise professionellen Mitwirkenden für ein lebendiges Musik- und Theaterleben zu nutzen vermochte.

Dass alle bisher erwähnten Sammlungen neben Geläufigem auch Singuläres und viel Hochkarätiges enthalten, sei festgestellt, kann und soll hier jedoch nicht erörtert werden. Denn der Blick ist zu lenken auf das quantitativ herausragende und auch inhaltlich bestimmende Bergmassiv im ,Mittelgebirge‘ der heutigen Dresdner Musik-Quellenbestände (um hiermit ein letztes Mal die geologische Metapher zu bemühen): auf das Musiziergut der 1548 gegründeten Hofkapelle der Kurfürsten und Könige von Sachsen. Obwohl seit 1897 nach und nach in die Königliche Öffentliche bzw. Sächsische Landesbibliothek gelangt und allgemein zugänglich gemacht, ist es bis heute noch viel zu wenig bekannt.

Sucht man nach den Ursachen für diesen Sachverhalt, so dürfte erstens eine Rolle gespielt haben, dass bis in die zweite Hälfte des 20. Jahrhunderts hinein das Hauptinteresse der Musikforschung den ,ganz Großen' der Musikgeschichte galt und entsprechende Arbeitsergebnisse teils in Monographien und Spezialuntersuchungen, teils in Gesamtausgaben und für die Praxis bestimmte Urtextausgaben von Werken dieser Meister einflossen. Zwar wurden auch örtliche und regionale Musikgeschichten sowie die sogenannten, Kleinmeister erkundet und deren Bereich des 
Musikerbes durch Denkmälerausgaben zugänglich gemacht. Aber zumeist führte das Bemühen erst einmal dahin, den Bedeutungsabstand zu besagten ,ganz Großen' mehr zu betonen als zu vermindern. Das änderte sich erst mit dem breiter werdenden Interesse an Alten Instrumenten, Alter Aufführungspraxis und dem entsprechenden Repertoire.

In diesem Zusammenhang hat es sich zweifellos negativ ausgewirkt, dass die Sächsische Landesbibliothek in den 1920er Jahren die Entscheidung traf, in der Musikabteilung ein einheitliches Signaturen-System und die auf diesem beruhende Regalaufstellung einzuführen. Das bedeutete, jeder Komponist erhielt eine Stammzahl; für die jeweilige Musikalie folgt danach ein Buchstabe, der die relevante Werkgattung (oder auch mehrere) bezeichnet, und schließlich eine hierauf bezogene laufende Zählung; Quellen mit Werken von mehr als einem Autor erhielten die Stammzahl 1, Anonyma die Zahl 2 (nach der Identifizierung des Komponisten jeweils von dessen Zahl abzulösen). ${ }^{1}$ Auf diese Weise wurden alte Überlieferungszusammenhänge und der Stellenwert der einzelnen Quelle unsichtbar gemacht. Wer nur wissen wollte, wie viele Autographen der genannten ,ganz Großen' in Dresden vorhanden waren, war von der Bedeutung der Dresdner Schätze kaum zu überzeugen. ${ }^{2}$

So lange die mit den Sammlungen ins Haus gekommenen Bestandsverzeichnisse existierten, ließen sich ehemalige Überlieferungszusammenhänge noch feststellen; seit dem Verlust von Verzeichnissen durch den Zweiten Weltkrieg ist das jedoch nur auf Umwegen möglich. Betroffen hiervon sind speziell die genuin Dresdner höfischen Sammlungen.

Nach dem Zweiten Weltkrieg hat die Bibliothek (und somit auch die Musikabteilung) dank ihres Alters, ihres Rufs als herausragende Fürstenbibliothek und dank einer hochengagierten Leitung ihren Sammelauftrag sowie ihre Erschließungs- und Vermittlungsarbeit nicht nur stets wahrnehmen, sondern sogar erweitern können, selbst unter den räumlichen Provisorien und den allgemeinen Erschwernissen dieser Jahrzehnte. ${ }^{3}$ Die Musikabteilung, bisher mit Bezug auf Katalogpublikationen völlig unterrepräsentiert, ${ }^{4}$ trat nun selbst mit der Publikation von Spezialkata-

1 Die dreiteiligen systematisch-numerischen Signaturen, ursprünglich für den Gesamtbestand der Musikabteilung bestimmt, gelten seit circa 1960 nur noch für den Quellenbestand der Musikalien (also nicht mehr für neue Musikdrucke und nicht für Musica theoretica jeden Alters). Zwischen Alten Drucken und Handschriften, zwischen Abschriften und Autographen wird mittels der Signatur jedoch nach wie vor nicht unterschieden. Bedingt durch Erfordernisse wie Möglichkeiten der EDV sind neuerdings lediglich die Bindestriche zwischen den Signaturteilen durch Punkte ersetzt worden, und identifizierte Anonyma erfahren keine Umsignierungen mehr, sondern behalten ihre, Zweier'-Signatur.

2 Die Verfasserin erinnert sich, noch Anfang der 1970er Jahre in der SLB eingehende Anfragen nach bestimmten Komponisten, speziell aus der Alte-Musik-Praxis, gelegentlich negativ beschieden, aber mit dem Hinweis auf ähnliches Vorhandenes, oft sogar Interessanteres zumeist lebhaftes Echo ausgelöst zu haben.

3 Die DDR-Regierung förderte im Bereich ihrer finanziellen Möglichkeiten zumindest den Bestandsaufbau und den Personalzuwachs der SLB erheblich; ein Mitbieten auf dem westeuropäischen Auktionsmarkt war devisenbedingt nicht möglich.

$4 \mathrm{Zu}$ nennen sind lediglich: Robert Eitner und Otto Kade, Katalog der Musik-Sammlung der Kgl. öffentlichen Bibliothek zu Dresden (im japanischen Palais), Leipzig 1890 (betrifft lediglich das vor den Zugängen der königlichen und der von auswärts stammenden Sammlungen Vorhandene), und Arno Reichert, „Musikalische Originalhandschriften“, in: Katalog der Handschriften der Sächsischen Landesbibliothek (vormals Kgl. Öff. Bibliothek) zu Dresden, Leipzig 1923, Bd.4, S. 195-250 (enthält nur den kleineren Teil des damals Vorhandenen, soweit der Überblick des Autors bis zum vorgegebenen Termin reichte; der Reprint von 1983 ist zumindest mit aktualisierenden Einträgen versehen). Die in Robert Eitners Quellenlexikon nachgewiesenen Bestände aus der König- 
logen, begleitenden Studien, mit fotomechanischen Nachdrucken alter Theoretika und sogar mit einer Faksimile-Reihe hervor ${ }^{5}$ und half aktiv, das Interesse an ihren Quellenschätzen zu wecken.

Nachteilig wirkten die politischen Ereignisse, besonders die Entstehung des Eisernen Vorhangs und seiner Folgen, sich dennoch aus. Nach der deutschen Wiedervereinigung nutzte die Sächsische Landesbibliothek sofort alle sich bietenden neuen Mittel, vor allem die Computertechnik und die Möglichkeit, Sonderaufgaben mit projektgebundenen Mitteln zu lösen. In Zusammenarbeit mit der im Haus stationierten RISM-Arbeitsstelle und zugleich DFG-gestützt kamen zwei von Ortrun Landmann vorgelegte Quellenkataloge zustande. ${ }^{6}$

Unter der Ägide der heutigen SLUB (1997 errichtet durch Zusammenschluss der SLB mit der TUB Dresden) und auf der Basis der neuen digitalen Techniken wurden und werden die Tiefenerschließung der Musikquellen fortgesetzt und der internationale Zugang zu ihnen erleichtert.

Vorrangig im Blick stehen hierbei zunächst die Kernbereiche, und das sind - herausragend durch Vielfalt, Umfang sowie inhaltliches und historisches Gewicht - die bereits erwähnten Überlieferungen der Dresdner Hofkapelle. Als übergeordnete Instanz der höfischen Musikpflege war die Kapelle verantwortlich sowohl für die Kirchenmusik als auch für Musik- und musikbegleitetes Theater sowie für das Repertoire von Kammer- und Orchesterkonzerten. Entsprechend vielseitig ist ihr Erbe - es könnte einzigartig sein, wenn es noch vollständig vorhanden wäre.

Der älteste Teil der Kapell-Musikalien - sicher nach der Zahl der Regalmeter ihrer historischen und kompositorischen Bedeutung weit unterlegen - fiel bereits 1760 der Beschießung Dresdens durch die preußische Armee zum Opfer. Die sogenannte „Instrumentkammer“ der Kapelle, zugleich Aufbewahrungsstätte des alten Notenfundus, wurde mit dem damaligen Prinzenpalais ${ }^{7}$ total zerstört. Da die zugehörigen Inventare wohl mitverbrannt sind, ist die alte Kapell-Sammlung kaum noch rekonstruierbar. Ihren Hauptinhalt bildeten zweifellos die Aufführungsmaterialien der Kapelle für alle Bereiche ihres Wirkens ab 1548 sowie die kompositorischen Hinterlassenschaften der Hofkapellmeister, darunter diejenige von Heinrich Schütz.

Vielleicht war 1760 in der Instrumentkammer zusätzlich auch frühestes Musiziergut aus der ersten katholischen Hofkirche (am Taschenberg, genutzt 1709-1750) zwischengelagert, vorgese-

lichen Privat-Musikaliensammlung verstreuen sich über alle Bände des Lexikons und sind häufig zu lapidar, um wirklich Auskunft geben zu können.

5 U.a. erschienen der von Wolfram Steude erarbeitete Katalog Die Musik-Sammelhandschriften des 16. und 17. Jahrhunderts in der Sächsischen Landesbibliothek zu Dresden, Leipzig 1974, die von der SLB im Eigenverlag herausgegebene Reihe Studien und Materialien zur Musikgeschichte Dresdens (1976-1987; sieben Hefte, darin ein erstes Werkverzeichnis zu Zelenka (Wolfgang Reich), ein Katalog der Telemann-Quellen sowie ein erster Katalog zur Opernüberlieferung (beide Ortrun Landmann), und die vom Zentralantiquariat Leipzig verlegte, von Ortrun Landmann konzipierte und betreute Faksimile-Reihe Musik der Dresdner Hofkapelle. Autographen und singuläre Abschriften (von 1980 bis 1987 erschienen 13 nicht nummerierte Bände).

6 Katalog der Hasse-Musikhandschriften in der SLUB Dresden (1999) und Das Dresdner Opernarchiv in der SLUB Dresden (2002), jeweils als CD-ROM-Katalog mit Begleitband, erschienen in München bei K.G. Saur.

7 Es handelt sich nicht um das Kur- oder Kronprinzen-Palais (als welches das Taschenberg-Palais diente), sondern um das für die Geschwister des Kurprinzen Friedrich Christian eingerichtete Prinzenpalais, welches in dieser Funktion nur relativ kurze Zeit zur Verfügung stand. An seiner Stelle, wenngleich auf verkleinertem Grundriss, wurde das sogenannte Landhaus errichtet, das heute als Stadt-Museum dient. Siehe Ortrun Landmann, „Topographische und aufführungspraktische Anmerkungen zu Hasses Dresdner Wirken“, in: Johann Adolf Hasse in seiner Zeit. Bericht über das Symposium vom 23. bis 26. März 1999 in Hamburg, hrsg. von Reinhard Wiesend, Stuttgart 2006, S. 317-342 (Hasse-Studien, Sonderreihe. 1). 
hen zur Aufarbeitung für die Weiterverwendung in der großen neuen Hofkirche von 1751. Hierbei müssten sich z.B. frühe Kirchenwerke Johann Adolf Hasses befunden haben, die in den Katalogen der neuen Kirche nicht auftauchen, also beim Anfertigen schon des ersten dieser Kataloge (1765) nicht mehr vorhanden waren. ${ }^{8}$

Es kann somit im Folgenden nur (und selbst hier nicht lückenlos) vom Repertoire aus der Zeit nach der Modernisierung der Hofkapelle die Rede sein. Die Modernisierung war eine Folge des 1696 vollzogenen Konfessionswechsels von August dem Starken ${ }^{9}$ zum Katholizismus, der diesen Schritt benötigte, um polnischer König werden zu können. Vielerseits bekämpft, rief August die Unterstützung des Papstes an; der Papst verlangte als Gegenleistung, dass der Wettiner seinen neuen Glauben öffentlich bekunde. Das hoffte dieser, am sinn- und effektvollsten mit der Kirchenmusikpflege tun zu können. Er hatte Erfolg damit, denn die hochkarätigen Kapell-Aufführungen bei Hochämtern und Festlichkeiten zogen Publikum an und halfen, die Ablehnung seines religiösen Schritts (bei dem auch Augusts Nachfahren verblieben) durch die lutherische Bevölkerung zu mildern.

Die Bedingungen dafür wurden geschaffen durch die Indienstnahme einer Gruppe Kapellknaben böhmischer Herkunft im Jahre 1708 sowie durch den Aufbau eines Orchesters aus den Hofkapell-Instrumentisten und hinzu engagierten Spielern damals modernster Instrumente. Interessanterweise hieß diese Formation nicht nur von Anfang an - also seit 1709/10 - „Orchester“, sondern sie besaß auch schon dessen Wesensmerkmale, denn sie bestand aus einem Quintett jeweils chorisch besetzter Violin-Instrumente sowie Paaren von Querflöten, Oboen, Fagotten und Hörnern. Die damals noch neuen Hörner dürften die frühesten in einem Orchesterverband überhaupt gewesen sein. ${ }^{10}$ Trompeten und Pauken standen zur Verfügung, bildeten aber eine besondere, nicht zum Kapell-Etat gehörige Gruppe. Vervollständigt wurde das Ensemble durch Sänger französischer und (zunehmend) italienischer Herkunft, und diese Sänger rechtfertigten die weiterhin geltende Bezeichnung des Gesamtkorpus als „Kapelle“. ${ }^{11}$ Ausgegliedert blieben - im Unterschied zum alten, evangelischen Kapellgefüge - die der Administration der Dresdner Jesuiten-Mission unterstellten katholischen Kapellknaben. ${ }^{12}$

Als stabilster Teil dieses Kapell-Konstrukts sollte sich das Orchester erweisen, während der vokale Bereich bereits nach circa 60 Jahren durch Ausgliederung der Opernsänger auf Kirchensänger reduziert wurde. ${ }^{13}$ Das Orchester durchlief trotz mehrmaliger Gefährdung (aber nie unterbrochener Existenz) glanzvolle Perioden, die es seit 1919 als Sächsische Staatskapelle fortsetzt.

8 Siehe Ortrun Landmann, „Fragen zu Johann Adolf Hasses Schaffen für die Dresdner Hofkirche am Taschenberg (1731-1750)“, in: Johann Adolf Hasse. Tradition, Rezeption, Gegenwart. Bericht über das Symposion vom 23. bis 25. April 2010 in der Hochschule für Musik und Theater Hamburg, hrsg. von Wolfgang Hochstein, Stuttgart 2013, S. 79-86 (Hasse-Studien, Sonderreihe. 3).

9 Lebenszeit 1670-1732; regierte als Kurfürst von Sachsen ab 1694 und zusätzlich als „König in Polen“ab 1697.

10 Nach Auskunft enzyklopädischer Artikel und einschlägiger Spezialarbeiten wurden Hörner in ihrer Frühzeit vor allem separat verwendet, vor allem in Verbindung mit fürstlichen Jagden.

11 Siehe Ortrun Landmann, „Die ,Geburt‘ des Orchesters innerhalb der Dresdner Hofkapelle“, in: Partita. Siebenundzwanzig Sätze zur Dresdner Musikgeschichte. Festschrift für Hans-Günter Ottenberg zum 65. Geburtstag, hrsg. von Wolfang Mende, Dresden 2012, S. 101-130.

12 Parallel dazu wurden die evangelischen Kapellknaben, die es weiterhin gab, dem evangelischen Konsistorium unterstellt.

13 Das geschah endgültig 1769. Spätere Aktivitäten von Opernsängern in der Kirche führten nur dann zur Kapellmitgliedschaft, wenn eine Berufung zum Kirchensänger erfolgte, und blieb auf diese Funktion beschränkt. 
Hinsichtlich der Kirchenaufgaben hatte es, wie dargelegt, um 1700 einen Schnitt gegeben. Von 1709 löste ein neues, dem katholischen Ritus verpflichtetes Repertoire das seit 1584 - mit allen stilistischen Weiterentwicklungen - gepflegte Repertoire des evangelisch-lutherischen Kirche ab, wiederum auf hohem bis höchstem Niveau. Erst mit der Aufhebung der Monarchie 1919 erlosch der Dienstsektor „Kirchenmusik“ für die Kapelle.

Dass nach dem ersten Jahrhundert der Existenz des Orchesters der Basso-Continuo-Apparat gänzlich verschwand und dafür die Instrumentenpalette Schritt für Schritt sowohl erweitert als auch modernisiert wurde, ergab sich aus dem allgemeinen Fortgang der Musikgeschichte. Das Fundament für die weitere Kapell-Entwicklung bildeten die Planstellen (die damals zwar nicht so hießen, es de facto aber waren). Jeder Planstelleninhaber, zugleich „Kammermusikus“, spielte nur ein Hauptinstrument, zu welchem zwar dieses oder jenes Neben-, aber kein weiteres Hauptinstrument hinzukam, so, wie noch heute z.B. das Englischhorn als Nebeninstrument von einem Oboisten mitversehen wird. Da die Stellenbewerber einer strengen Auswahl unterzogen wurden und, von Ausnahmen abgesehen, bis zum altersbedingten Ausscheiden im Amt blieben, war sehr rasch höchste Qualität erreicht und wurde dann nahezu durchgängig gehalten.

Dem Dresdner Kapell-Orchester oblagen dafür auch enorme Pflichten. Wegen der drei generellen Wirkungsbereiche katholische Kirche (über lange Zeit mit fast täglichen Diensten), Kammer (d.h. Musizieren an Örtlichkeiten, die dem höfischen Publikum vorbehalten waren) und Theater (wo zu Opern- und Ballettaufführungen auch Schauspielmusiken und Ähnliches kamen) gab es stets mehrere Kapellmeister. ${ }^{14}$ Da diese bis 1860 mit Komponierverpflichtung angestellt wurden, konnte für Kirche und Theater der Bedarf an fremden Werken zunächst entfallen oder doch eingeschränkt bleiben. Für die Kammermusik lag die Hauptverantwortung hinsichtlich der Repertoire-Beschaffung bei den Konzertmeistern; ${ }^{15}$ darüber hinaus sorgte jeder Solospieler für sein eigenes Repertoire. Hier herrschte daher von Anfang an Vielfalt bei der Herkunft der Werke.

Der Hof bezahlte für Kirchen- und Theatermusik das Stimmenmaterial; die Partituren blieben Eigentum ihrer Autoren, bis man sie ankaufte - oft erst nach deren Tod, als Nachlass. Waren Partituren (nicht nur von fremden, sondern auch von eigenen Komponisten) bereits abschriftlich vorhanden, wie vor allem bei Johann Adolf Hasse, so bestand an den Autographen später kein Interesse mehr. Ob mit oder ohne Partitur: Das Aufführungsmaterial wurde als höfisches Eigentum aufbewahrt. ${ }^{16}$

Anders verhielt es sich, wie gesagt, mit der Kammermusik. Dass hier ein in jüngster Zeit wieder in den Blick der Öffentlichkeit geratener Fundus überlebt hat, ist mehreren Ursachen zu verdanken: zunächst dem Umstand, dass die Königin Maria Josepha (Schwiegertochter Augusts des Starken) die Sammlung des Konzertmeisters Johann Georg Pisendel (1687-1755) aus dessen Nachlass angekauft hat. Als die durch den Siebenjährigen Krieg bedingte Notlage zu äußerster Sparsamkeit zwang und dennoch dem Kapell-Orchester mehr Abwechslung bei den in der Kirche zu spielenden Instrumental-Pieçen verschafft werden sollte, wurde 1774 ein Schrank hinter der Hofkirchenorgel aufgestellt und dort die Pisendel-Sammlung eingelagert. Diese erhielt zwecks geordneter Nutzbar-

14 Als solche wirkten im Kirchenbereich auch die rangniedrigeren „Kirchen-Compositeur““.

15 Zwei Konzertmeister (1. Violine) gab es ab 1817.

16 Wenn der Komponist ausschließlich selbst die Aufführungen aus seiner eigenen Partitur leitete und diese nicht nachträglich erworben wurde, so fehlt sie bis heute. 
keit einheitliche Umschläge mit einheitlicher Titelbeschriftung und einen dazu gehörigen Katalog. ${ }^{17}$ Damit war der „SchranckNo. II.“ geboren. So manches daraus blieb, gleich vielen für die Hofkirche geschaffenen Sakralwerken, über kürzere oder längere Zeit Bestandteil der dortigen Musikpflege.

In einer Epoche, die noch nach immer neuer Musik verlangte und Älteres ,altmodisch ' fand, dürfte dies als sehr frühes Beispiel einer sich bildenden Repertoire-Tradition anzusehen sein. Diese Tradition brachte Vorteile, denn die Musiker, die in Theater und „Kammer“ ständig Novitäten darzubieten hatten, werden es als Erleichterung erlebt haben, in der Kirche auch ihnen wohlbekannte Werke vortragen zu dürfen, die zudem von Aufführung zu Aufführung an Perfektion noch gewinnen konnten. ${ }^{18}$

Notensammlungen von Pisendels berühmten Kapellkollegen sind hingegen nicht erworben worden, vor allem wohl, weil die Sammlerin Maria Josepha schon 1757 verstarb. Vereinzelte ältere Kollektionen - nachweislich diejenige des Konzertmeisters Jean-Baptiste Woulmyer (circa 16771728) - verbrannten 1760 in der Instrumentkammer. ${ }^{19}$ Spätere Wettiner musizierten sehr eifrig selbst, sammelten Musikalien demgemäß für ihren eigenen Bedarf und vereinten sie mit dem Familienerbe zur Königlichen Privat-Musikaliensammlung (KPMS). Die Betreuung dieser Sammlung wurde, analog den kapellpraktischen Sammlungen, einem Kammermusiker übertragen. Der Flötist Moritz Fürstenau, ${ }^{20}$ bekanntester KPMS-Custos, vermehrte diese Sammlung erheblich, indem er aus dem Kirchen- und dem Opernarchiv herüberholte, was er als besonders kostbar erkannte bzw. was von der Praxis inzwischen ausgesondert worden war. ${ }^{21}$ Hierdurch erreichte u. a. der Inhalt von Schrank II die nächste Stufe seines Bewahrtbleibens. Mit der gesamten KPMS gelangte er auf königliche Anweisung 1897 in die Königliche Öffentliche Bibliothek.

Allerdings hatte Fürstenau seinem Traum, die KPMS zu einem Archiv europäischer Musikgeschichte auszubauen, kostbare Dresdner Besitzstücke im Tausch gegen neu angefertigte Abschriften von auswärtigen Quellen geopfert. Noch immer zählte der Gebrauchswert - zu praktischen wie nun auch zu musikhistorischen Zwecken - mehr als die unersetzliche originale Überlieferung. ${ }^{22}$

17 Dieser vermutlich 1945 verbrannte Katalog trug laut Repertorium des Bibliotheksarchivs den Titel: Catalogo derer Musicalien in dem Schranke No II, worin Solo, Trio, Concerten, Ouverturen und Pieçen sowohl vor die Cammer als Teatre sich befinden. 1774. Das erweist die Sammlung als ursprünglich nicht für die Kirche bestimmt. Interessanterweise sind für kirchliche Aufführungen nur Stimmen von ersten Sätzen vervielfältigt worden: diese Sätze genügten als Gradualmusiken.

18 Nicht einmal Opern waren um jene Zeit ,langlebig; sie verschwanden rasch vom Spielplan.

19 Die Woulmyer-Sammlung, wohl vorwiegend französische Werke umfassend, mag als veraltet angesehen und sofort, archiviert' worden sein.

20 Lebenszeit 1824-1889. Moritz folgte seinem 1852 verstorbenen Vater 1853 in dieser Funktion. Anton Bernhard Fürstenaus Vorgänger scheint der als Gesangslehrer berühmte Johannes Miksch gewesen zu sein; dessen Vorgänger müssen noch ermittelt werden.

21 Da ohnehin königliches Eigentum, war die Einordnung in die KPMS ohne bürokratischen Aufwand möglich. Eine Eingabe Fürstenaus an den König vom November 1860 (D-Dl: Bibl.Arch.III.Hb,Vol.800.a) wurde offenbar positiv beschieden und bildete somit den Auftakt für die umfangreiche Aktion, die u.a. die Übernahme des gesamten Schrank-II-Bestandes einschloss.

22 Ein Beispiel für weitere bildet das Autograph der Violinsonate RV 19 von Antonio Vivaldi, eines von ehemals mindestens fünf, die Vivaldi Pisendel geschenkt hatte. Auf welchem Wege es aus Dresden in die Sammlung Malherbe und von dort in die Bibliothèque nationale de Paris gelangte, ist noch unerforscht. Siehe die Faksimile-Ausgabe in der von Ortrun Landmann herausgegebenen Reihe Musik der Dresdener Hofkapelle, Leipzig 1982 (Bandkommentar von Karl Heller). 
In Dresden war ja so vieles in Mehrfachexemplaren vorhanden, und so kam es z.B. um 1854 mittels königlicher Verfügung zu einer umfangreichen Schenkung an das Leipziger Konservatorium. ${ }^{23}$ Die Abgabe sogenannter „Doubletten“ (eine Klassifikation, die auf Handschriften gar nicht angewendet werden darf!) hatte bereits im 18. Jahrhundert begonnen; wie lange sie fortgesetzt wurde, ist noch so unbekannt wie deren gesamtes Ausmaß.

Unglücklicherweise wurde bei den zurückbehaltenen Handschriften nicht nach deren Alter und Überlieferungswert gesehen, sondern nach einem möglichst sauberen, von Einträgen freien Schriftbild und nach möglichst gutem Einband. So ging nach und nach Wesentliches verloren. ${ }^{24}$

Den bisher - und hoffentlich überhaupt - letzten Verlust, zum zweiten Mal katastrophalen Ausmaßes, brachten der Zweite Weltkrieg und seine Folgen. Besonders hart traf es die umfangreichen Hofkirchen-Bestände, die, nach ihrer Übernahme 1908, ${ }^{25}$ von der SLB bis 1945 erst zu einem kleinen Teil eingearbeitet worden waren. Immer noch einzig vom Aspekt des praktischen Nutzens geleitet, hatte die Bibliothek allen Musikbesitz zu einer durchsystematisierten Sammlung zusammenführen wollen, verfügte aber über viel zu wenig Personal und wich den Schwierigkeiten beim Katalogisieren und Einkapseln der Stapel von Stimmenmaterialien aus, indem sie deren Bearbeitung zugunsten von Partituren hintanstellte. Die nicht katalogisierten, ungestempelten Stimmenstapel sind 1945 zwar nicht verbrannt, wurden nach aller Wahrscheinlichkeit aber 1946, gleich erheblichen Mengen an ausgelagertem Bibliotheksbesitz, in die Sowjetunion abgeführt, wo ihr Schicksal kaum noch zu verfolgen ist.

So hat die schrittweise Zentralisierung des historischen Musizierguts der Hofkapelle, ehemals vorhanden in reicher Fülle und in allen seinen Facetten, letzten Endes auch zu Schäden und Einbußen geführt. Relativ gut davongekommen ist zufälligerweise der Opernarchiv-Bestand, ${ }^{26}$ denn

23 Siehe Maren Goltz, Studien zur Geschichte der Bibliothek der Hochschule für Musik und Theater „Felix Mendelssohn Bartholdy“ Leipzig von 1843 bis 1945. Mit einem Ausblick bis zur Gegenwart, Berlin 2003. Darin S. 25: „Es handelte sich um 262 Bände Partituren, Klavierauszüge bzw. Stimmen, überwiegend in Form von Abschriften und Erstdrucken.“ Der Aktenband 10711 (Min. des Kgl. Hauses), Loc.48 Nr.4 in D-Dla enthält das von den Empfängern im Januar 1855 quittierte Verzeichnis der Schenkung mit allein 51 Werken Johann Adolf Hasses in 98 Bänden.

24 Bezüglich der heutigen Streuung von ehemals in Dresden befindlichen Hasse-Partituren und deren Quellenrang gewinnt man einen ersten Überblick bei Roland Dieter Schmidt-Hensel, „La musica è del Sig. Hasse detto il Sassone ...“. Johann Adolf Hasses „Opere serie“ der Jahre 1730 bis 1745. Quellen, Fassungen, Aufführungen, 2 Bde., Hamburg 2004. Wann und auf welchem Wege die Handschriften ihre jetzigen Besitzer fanden, wird in der Arbeit nicht untersucht. Von besonderem Interesse wäre es zu erfahren, wann und wo François-Joseph Fétis in den Besitz von Dresdner Kopialien gelangte. Nicht auszuschließen ist hier die Auktion der Breitkopfschen Stammabschriften Leipzig 1834. Siehe dazu u.a. Ulrich Leisinger und Peter Wollny, Die Bach-Quellen der Bibliotheken in Brüssel, Hildesheim u.a. 1997; auch Ortrun Landmann, „Breitkopf's Music Trade as Reflected in the Holdings of the Sächsische Landesbibliothek“, in: J.S. Bach, the Breitkopfs, and Eighteenth-Century Music Trade, hrsg. von George B. Stauffer, Lincoln u.a. 1996, S. 169-179 (Bach Perspectives. 2). Ein besonderes Kapitel bildet das ,Abschöpfen' der Dresdner Sammlungen, einschließlich ihrer als überholt ausgesonderten Kataloge, durch Georg Poelchau. Sein Sammelgut befindet sich heute in D-B.

25 Die Übernahme schloss damals noch in Benutzung Befindliches (darunter Partitur-Autographen, aus denen dirigiert wurde!) aus. Hiervon haben nur Fragmente überlebt; sie kamen 1972ff. in die SLB.

26 Diese Sammlung wurde erst nach dem Siebenjährigen Krieg begonnen, genauer mit der Impresa Bertoldi (ab 1780); von der vorangehenden Impresa Bustelli liegen nur die zusätzlich für den Hof gefertigten, in der KPMS überlieferten Partitur-Kopien vor. Siehe Ortrun Landmann, Die Dresdner italienische Oper zwischen Hasse und Weber. Ein Daten- und Quellenverzeichnis für die Jahre 1765-1817, Dresden 1976. 
dessen Hauptmasse gelangte erst 1955, also zu DDR-Zeiten, in die SLB, nachdem sie Krieg wie Nachkrieg zwar nicht unbeschadet, aber glimpflich überstanden hatte.

Teilweise katastrophale Früh- und Spätfolgen brachte nach den Bombenangriffen 1945 das wochenlang unentdeckte Liegen im Wasser für Kostbarkeiten der Bibliothek, darunter auch für solche aus dem Schrank-II-Bestand. An diesen Opfern konnte sich immerhin die hauseigene Papierrestaurierungswerkstatt zu immenser Leistungshöhe entwickeln und so den Hauptteil retten.

Überhaupt gelang der SLB zu besagten DDR-Zeiten eine unter den gegebenen Bedingungen unglaubliche Wiederaufbauarbeit an ihrem dezimierten Bestand. Darüber hinaus begann nun auch, vor allem in der Musikabteilung, eine Quellenerschließung, angeregt durch das internationale Wirksamwerden von RISM und durch Arbeiten einzelner Forscher (immer wieder zu nennen ist hier der Initialanstoß, den die Untersuchungen von Karl Heller zur Überlieferung der Vivaldi-Handschriften ${ }^{27}$ gegeben haben). ${ }^{28}$

Wirkliche ,Forschung' mit allen nötigen Zusatzermittlungen auch außer Haus konnte nicht geleistet werden, ${ }^{29}$ das sah ein Bibliotheks-Arbeitsprogramm damals so wenig vor wie heute. ${ }^{30}$ Aber der Segen der Zusammengehörigkeit großer Bestandsgruppen zeigte sich nun endlich und unmittelbar an diesen selbst, nämlich anhand der Möglichkeit, Einzelstücke und sogar versprengte Stimmen einander zuzuordnen aufgrund ihrer Schreiber, Papiere, Titelaufschriften. Eine andere Möglichkeit hätte es infolge der 1945 offenbar verbrannten, ehemals zahlreichen alten Kataloge und Spezialverzeichnisse auch gar nicht mehr gegeben. Der Erkenntnisprozess verlief durchaus mit Um- und sogar Abwegen, führte aber auch zu Ergebnissen, die voraussichtlich Bestand haben werden.

$\mathrm{Zu}$ verdanken ist dies vor allem der Tatsache, dass es bei der Dresdner Kapelle Planstellen für professionelle Notisten gab (NB: „Notisten“ war ihre amtliche Bezeichnung, im Unterschied zu Kanzlei-Kopisten, die buchstabengetreu Worttexte zu kopieren hatten, ohne korrigierende Eingriffe, wie sie von Notisten sogar erwartet wurden; der Notist war dem Kopisten somit an Qualifikation weit überlegen). Die Dresdner Notisten, nachweisbar seit etwa Mitte des 17. Jahrhunderts, ${ }^{31}$ entwickelten deutliche, den meist schlechten Beleuchtungsverhältnissen beim Musizieren angemessene, gebrauchsgerechte Schriftformen. Da sie außerdem für den Privatbedarf der Wettiner schrieben, erfüllten ihre Erzeugnisse zumeist auch bemerkenswerte ästhetische Ansprüche. Betont sei, dass es sich dabei nicht um Prachthandschriften handelt, angefertigt zum Zweck des Sammelns, sondern durchweg um Gebrauchsgut für die Praxis, bei der Kapelle wie bei den Wettinern. ${ }^{32}$

27 Im Druck erschienen Leipzig 1971.

28 So kamen allmählich Zusammenhänge wieder in den Blick, die durch die um 1930 vorgenommene Bestandsvereinheitlichung mittels der bekannten dreiteiligen Signaturen und durch den hohen Verlust an alten Katalogen nicht mehr präsent waren.

29 Ausnahme: der Oelser Dittersdorf-Bestand, zu welchem ein Katalog und ergänzende Aufsätze von Ortrun Landmann vorgelegt wurden.

30 Die inzwischen mögliche Nutzung von DFG-Sondermitteln für einzelne Erschließungsprojekte bedeutet zwar einen großen Schritt nach vorn, ist allerdings kein Allheilmittel, da die Einarbeitungszeit, die jeder Projektbearbeiter für sich selbst benötigt, von jener Zeit abzuziehen ist, die für das Vorlegen fertiger Ergebnisse nötig wäre. Das wird bei derartigen Projektplanungen leider übersehen.

31 Über die Schaffung des Notisten-Amts (vorerst mit einer bzw. zwei halben Stellen) und seine ersten Inhaber ist noch nichts Konkretes ermittelt.

32 Natürlich gehören zum Bestand der KPMS auch Prachthandschriften und -drucke; sie gelangten z.B. als Widmungsgaben ihrer Autoren dorthin, sind aber für die KPMS nicht typisch. 
Und somit lässt sich ein drittes Charakteristikum der Dresdner königlichen Musikalien feststellen, neben ihrem Status als Primärsammlungen und neben ihrer Praxis-Gebundenheit: ihre durch identische Schriften gegebene Komplexität.

Das alles unterscheidet sie von Sekundärsammlungen, die meist mit historischem Abstand und meist von spezielle Aspekte verfolgenden Privatsammlern zusammengetragen wurden. Solchen Sammlern, deren Tun gar nicht überschätzt werden kann, verdanken wir die Erhaltung groBer und sogar wichtigster Teile des gesamten Musikerbes. Durch zumeist käufliche Übernahme in große Bibliotheken wurde deren Sammlungen dann die nach menschlichem Ermessen endgültige Bewahrung gesichert.

Welche Problemfülle aber durch Sammler zusammengetragene Einzelquellen in sich bergen können, hat wohl zuerst die Bach-Forschung deutlich gemacht: Es erforderte langwierige, international geleistete Arbeit, um die ihrem Umfeld entrissenen Quellen in ihren historischen Kontext wieder einordnen zu können.

Anders bei den gewachsenen Sammlungen, umgeben von dem sie dokumentierenden Material! Solches Material bieten für die Dresdner königlichen Sammlungen vor allem die im Sächsischen Hauptstaatsarchiv verwahrten Hofkapell-Akten. ${ }^{33}$ Sie sind zuverlässige Hilfen beim einander Zuordnen von Schriften und Schreibernamen sowie beim Datieren. Das aber ist inzwischen bekannt und soll hier nicht wiederholt werden.

Schwierigkeiten brachten und bringen weiterhin einige Notistenschriften, die erhebliche Veränderungen durchlaufen haben, und hier Erkenntnislücken zu schließen, ist eine noch keineswegs beendete Aufgabe. Hierfür im Folgenden zwei Einzelbeispiele.

Erstens bemerkte kürzlich die Verfasserin bei zufälligem Blick auf einige Hofkirchen-Reststimmen, dass daran außer Johann Gottfried Grundig auch Johann Gottlieb Morgenstern beteiligt ist. ${ }^{34}$ Morgenstern, dessen ausgereifte Schrift einst von Karl Heller mit dem Kennbuchstaben „D“ bezeichnet worden war, ist nie Hofnotist gewesen; er war 1722 als Kapell-Bratschist angestellt worden, arbeitete nebenher aber wohl bis mindestens 1750 als Lohnnotist und ist für die Kapell-Überlieferung jener Zeit nahezu so wichtig gewesen wie die beiden Amtsträger Johann Gottfried Grundig und Johann George Kremmler (I). Morgensterns Schriftentwicklung zählt zu den kompliziertesten überhaupt, ja, sie ist chamäleonartig, und entsprechend im Zickzack verlief und verläuft noch immer der Prozess ihrer Identifizierung. Jetzt, wo die große Linie dieser Entwicklung erkannt zu sein scheint, ${ }^{35}$ fügen sich nicht nur viele Details gut ein, sondern es wird Zusätzliches sichtbar. So darf bei den eben genannten Stimmen, die zu einem Confitebor von

33 Nicht zu unterschätzen sind auch die (meist gedruckten) Saxonica in D-Dl und D-Dla.

34 Auf S. 148 der Drei Studien 2010 (wie Anm. 38) wurden von der Verfasserin noch alle vier Vokalstimmen, verwahrt unter der Signatur Mus.2398-E-511 (Heinichen, Confitebor SeiH 31), Johann Gottfried Grundig zugewiesen und später datiert. Doch die Stimme Basso stammt von Morgenstern. Da nur sie, wie das Partiturautograph, die „No. 31“ über dem Beginn trägt, ist sie wohl direkt von diesem abgeschrieben worden, sehr wahrscheinlich 1727. Bei den Grundig-Stimmen, die zudem auf anderem Papier notiert sind, fehlt die Nummer. Über Gleichoder Ungleichzeitigkeit der Entstehung der beiden Schreibarbeiten soll hier nicht spekuliert werden; auf jeden Fall geht die Morgenstern-Stimme auf das Autograph zurück und lässt sie als zum originalen Stimmensatz gehörig vermuten.

35 Siehe Landmann, Zu den Dresdner Hofnotisten des 18. Jahrhunderts. Sieben Thesen und ein Anhang, Dresden 2014. Volltext auf Qucosa: http://nbn-resolving.de/urn:nbn:de:bsz:14-qucosa-144502. 
Johann David Heinichen gehören, die von Morgenstern stammende Basso-Stimme wohl ebenso wie die datierte autographe Partitur für 1727 angesetzt werden. Das wäre ein erster Nachweis dafür, dass Morgenstern bereits damals Lohnarbeiten für die Kapelle ausgeführt hat, während bisher von ihm aus jener Zeit nur seine privaten Schreibleistungen für Pisendel bekannt waren.

Eine zweite Neubetrachtung könnte sogar zu Resultaten führen, die über Schreiber und Schreibedatum hinausreichen. Es geht um die Fagottkonzerte der Pisendel-Sammlung, die nicht nur kürzlich ediert, sondern inzwischen auch auf CD eingespielt worden sind. Ihre Komponisten heißen Antonín Reichenauer, Franz Horneck und Graun (Vorname ungenannt). Bisher lag die Annahme nahe, dass die Noten sämtlich von Antonín Möser mitgebracht worden sind, als dieser 1737, nach dem Tod des Grafen Morzin, von Prag nach Dresden kam und hier in die Hofkapelle aufgenommen wurde. Schon die Papiere der Fagottkonzerte von Graun und von Reichenauer (C-Dur-Konzert) ${ }^{36}$ lassen kaum bezweifeln, dass diese beiden Kopien hier in Dresden entstanden sind. Mutmaßlich geschah dies bereits etliche Jahre vor Mösers Ankunft: Die Graun-Partitur ${ }^{37}$ nämlich zeigt die sorgfältige Schrift Grundigs in der „A1“-Form, die Reichenauer-Stimmen sind wohl Morgenstern zuzuweisen in einem Stadium, das dessen Schrift auf dem Wege der Annährung an Grundig zeigt. ${ }^{38}$ Es ist nicht bekannt, dass schon damals, d.h. in der zweiten Hälfte der 1720er Jahre, Möser in Dresden gastiert hätte oder ein Kapell-Fagottist als Virtuose hervorgetreten wäre.

Als des Rätsels Lösung sei hier, sehr verknappt, folgende Hypothese angeboten. Antonín Möser scheint der früheste Fagottvirtuose in der Musikgeschichte überhaupt gewesen zu sein. Sein Dienstherr Morzin dürfte Antonio Vivaldi, der in der Widmung seines Opus 8 eine Art Dienstverhältnis zu Morzin bestätigt, Anfang der 1720er Jahre ersucht haben, Solo-Konzerte seines neuen Stils für Möser zu komponieren, was Vivaldi offensichtlich befolgte. ${ }^{39}$ Morzin wie Möser ihrerseits haben ähnliche Konzerte dann wohl bald auch von weiteren Musikern erbeten, so von dem in Prag lebenden Antonín Reichenauer und von Franz Horneck, dem man in Prag oder vielleicht in Italien begegnete (über diesen Musiker ist ohnehin zu wenig bekannt). „Graun“ schließlich könnte sich als der Geiger Johann Gottlieb erweisen, der ja u.a. Schüler Tartinis gewesen sein soll. Freilich muss er zu diesem Zweck nicht nach Padova gereist sein, denn Tartini hielt sich von 1723 bis 1726 in Prag auf, ${ }^{40}$ und hierher kamen 1723 anlässlich der Königskrönung von Kaiser Karl VI. bekanntlich zahlreiche Musiker - unter ihnen wahrscheinlich der ältere Graun. Hier wäre dann dessen Kontaktaufnahme auch zum Grafen Morzin zwangsläufig gewesen. Die Rückreise gen Norden müsste Graun (wohl infolge der Abreise Tartinis von Prag) 1726 angetreten haben mit Nahziel

36 D-Dl: Mus.2474-O-47 bzw. Mus.2494-O-1.

37 Am Ende mit dem Monogramm „IGG“ versehen.

38 Eine ähnliche Form siehe Abb. III.135 und III.136 in Ortrun Landmann, Über das Musikerbe der Sächsischen Staatskapelle: drei Studien zur Geschichte der Dresdner Hofkapelle und Hofoper anhand ihrer Quellenüberlieferung in der SLUB Dresden, Dresden 22010, http://nbn-resolving.de/urn:nbn:de:bsz:14-qucosa-38515, S. 448f.

39 Zum Verhältnis Vivaldi-Morzin siehe, außer Vivaldis Widmungstext zum Druck seines Opus 8 (Il Cimento dell'Armonia e dell'Inventione, Amsterdam 1725), Karl Heller, Die deutsche Überlieferung der Instrumentalwerke Vivaldis, Leipzig 1971, sowie den Aufsatz von Michael Talbot, „Wenzel von Morzin as a Patron of Antonio Vivaldi“, in: Fasch-Studien IX, Beeskow 2003, S. 67-76. Die Vivaldi-Forschung vermutete allerdings bisher eine etwas spätere Entstehung der Fagottkonzerte und brachte sie nicht explizit mit Möser in Verbindung.

40 Siehe Artikel „Tartini“, in: MGG2, Personenteil Bd. 16, Kassel u.a. 2006, Sp. 522-532. Siehe auch Pisendels Brief an Telemann von 1750, worin Tartinis Anwesenheit in Prag bestätigt wird (Telemann, Briefwechsel. Sämtliche erreichbare Briefe von und an Telemann, hrsg. von Hans Grosse und Hans Rudolf Jung, Leipzig 1972, S. 355, Zeile 3). 
Dresden, wo er seinem alten Förderer Pisendel seine Prager Ausbeute an Erlerntem und Komponiertem vorlegte und ihm Abschriften verehrte, z.B. von seinem eigenen Fagottkonzert und vielleicht von dergleichen aus anderer Feder. Wohl dank Pisendels Vermittlung erlangte Graun dann bald seine Anstellung in Merseburg. Dies alles sind vorerst Vermutungen, aber von jener Art, die die Wichtigkeit von Handschriftendatierungen für das Erkennen historischer Zusammenhänge unterstreicht, besonders innerhalb bekannter Umfelder.

Zum Schluss noch eine weitere Hypothese, die zwar keine Handschrift betrifft, wohl aber die Identifizierung des Inhalts einer gedruckten Quelle mit dem einer Handschrift nahelegt, welche sich einst in Dresden befunden haben dürfte. Wie erwähnt, fehlen in Dresden die Notennachlässe so mancher Kapellmusiker, die einst für ihr Instrument Wesentliches geleistet haben. Es ist aber möglich, einiges daraus in anderweitiger Überlieferung wiederzufinden, denn die Kapelle und ihr Repertoire besaßen eine weitreichende Vorbildwirkung. So enthält die Sammlung Wenster im schwedischen Lund zeitgenössische Abschriften von frühem Dresdner Horn-Repertoire, das hier am Ort nicht mehr vorhanden ist. ${ }^{41}$

Ein weiteres ehemals für Dresden bestimmtes Werk lässt sich zudem vermuten im Concerto für zwei Hörner TWV 52:Es1 von Georg Philipp Telemann, enthalten in der 3me Production seiner Musique de table, gestochen und publiziert 1733. Ist schon ein Horn-Doppelkonzert nicht alltäglich, ${ }^{42}$ so fällt hier zudem die große viersätzige Gesamtanlage auf. Sie erinnert an das sogenannte „Concerto grosso“ für Violine und Orchester TWV 51:B1, das Telemann 1719 in Dresden für Pisendel schrieb und das dieser mit der Kapelle im Rahmen der damaligen Kurprinzen-Hochzeitsfeierlichkeiten aufgeführt hat. ${ }^{43}$ Bei dem Horn-Doppelkonzert spricht für den nämlichen Entstehungsanlass - deutlicher noch als die Viersätzigkeit - die an den Beginn gesetzte Polonaise. Sie galt damals als das musikalische Symbol für das polnische Königtum und wurde von den Dresdner Komponisten, einschließlich Johann Sebastian Bach, ${ }^{44}$ sogar in die Kirchenmusik übernommen als Anspielung auf das göttliche Himmelskönigtum. Es ist denkbar, dass die Dresdner Hornisten Johann Adam Schindler ${ }^{45}$ und Johann Adam Franz Samm Telemanns Partitur damals zum Geschenk erhielten. Samm starb 1723; zehn Jahre später, als Schindler, der hohe Hornist, den Beruf wechseln musste, könnte Telemann ihn um Rückgabe des Concerto gebeten haben, und die Veröffentlichung noch im Jahre 1733 wäre plausibel.

41 Hierauf machte Peter Damm, Solohornist der Sächsischen Staatskapelle, die Verfasserin dankenswerterweise bereits vor 30 Jahren aufmerksam. Damals übersandte die UB Lund der SLB als Geschenk auch Kopien der Wenster-Abschriften.

42 D-Dl besitzt aus der Pisendel-Sammlung zudem die Konzerte TWV 52:D2 und 52:Es1 (Mus.2392-O-27 bzw. -O-31).

43 Siehe die neben der autographen Partitur (Mus.2392-O-38) vorliegenden, von Pisendel selbst in sichtbarer Eile ausgeschriebenen Stimmen (Mus.2392-O-58), die auf praktische Nutzung deuten.

44 Siehe Szymon Paczkowski, Styl polski w muzyce Johanna Sebastiana Bacha, Lublin 2011 (Studia et dissertations Instituti Musicologiae Universitatis Varsoviensis. 16).

45 In ihrem Booklet-Text zur Einspielung dieses Concerto und dreier Fagottkonzerte durch die Dresdner Kapellsolisten (Ars Production, erschienen 2016) ging die Verfasserin noch irrtümlich davon aus, dass Johann Adalbert Fischer und Johann Adam Franz Samm, die ersten Dresdner Hornisten, das Horn-Doppelkonzert aus der Taufe gehoben hätten. Fischer hatte die Kapelle aber bereits vor den Festwochen von 1719 verlassen und war umgehend durch Johann Adam Schindler ersetzt worden. Eine diese Personalfragen einschließende Arbeit der Verfasserin befindet sich in Vorbereitung. 
Abschließend ein kleiner Appell an diejenigen Fachkollegen, die dergleichen Schlussfolgerungen als ,Spinnerei' ansehen: Die Verfasserin gesteht, dass sie das Spinnen für oft unvermeidlich hält, um bei der Lösung von Quellen-Problemen auf einen begehbaren Weg zu gelangen. Doch selbst bei einer historisch gewachsenen Sammlung wie der hier vorgestellten sollte man stets damit rechnen, dass Erkenntnis-Hypothesen durch neu auftauchende Aspekte ein ,update erfahren oder sogar durch feste Tatsachen umgestoßen werden. Insofern bieten zeitlich begrenzte, fremdfinanzierte Forschungsprojekte zwar eine chancenreiche Erschließungshilfe, erübrigen aber nicht generell die langjährig fortgesetzte Beschäftigung mit der jeweiligen Materie. 
